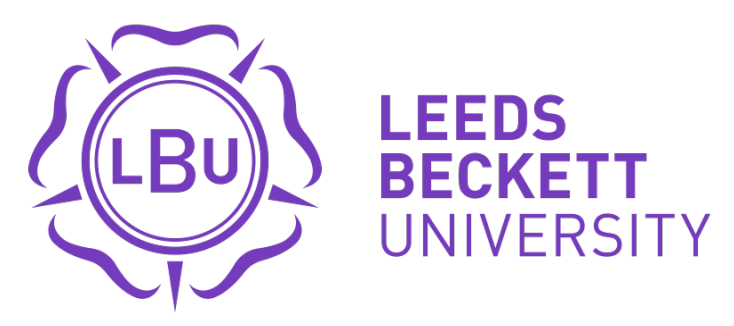

Citation:

Gerodetti, N and Besnard, A (2016) Ann Laura Stoler: La chair de l'empire. Savoirs intimes et pouvoirs raciaux en régime colonial. Nouvelles Questions Feministes, 35 (2). pp. 125-128. ISSN 0248-4951 DOI: https://doi.org/10.3917/nqf.352.0125

Link to Leeds Beckett Repository record:

https://eprints.leedsbeckett.ac.uk/id/eprint/4297/

Document Version:

Article (Published Version)

Creative Commons: Attribution 4.0

The aim of the Leeds Beckett Repository is to provide open access to our research, as required by funder policies and permitted by publishers and copyright law.

The Leeds Beckett repository holds a wide range of publications, each of which has been checked for copyright and the relevant embargo period has been applied by the Research Services team.

We operate on a standard take-down policy. If you are the author or publisher of an output and you would like it removed from the repository, please contact us and we will investigate on a case-by-case basis.

Each thesis in the repository has been cleared where necessary by the author for third party copyright. If you would like a thesis to be removed from the repository or believe there is an issue with copyright, please contact us on openaccess@leedsbeckett.ac.uk and we will investigate on a case-by-case basis. 


\title{
ANN LAURA STOLER : LA CHAIR DE L'EMPIRE. SAVOIRS INTIMES ET POUVOIRS RACIAUX EN RÉGIME COLONIAL
}

\author{
Natalia Gerodetti, Agathe Besnard
}

Editions Antipodes | « Nouvelles Questions Féministes »

2016/2 Vol. 35 | pages 125 à 128

ISSN 0248-4951

ISBN 9782889011278

Article disponible en ligne à l'adresse :

https://www.cairn.info/revue-nouvelles-questions-feministes-2016-2-page-125.htm

\section{Pour citer cet article :}

Natalia Gerodetti, Agathe Besnard « Ann Laura Stoler : La chair de l'empire. Savoirs intimes et pouvoirs raciaux en régime colonial », Nouvelles Questions Féministes 2016/2 (Vol. 35), p. 125-128.

DOI 10.3917/nqf.352.0125

Distribution électronique Cairn.info pour Editions Antipodes.

(C) Editions Antipodes. Tous droits réservés pour tous pays.

La reproduction ou représentation de cet article, notamment par photocopie, n'est autorisée que dans les limites des conditions générales d'utilisation du site ou, le cas échéant, des conditions générales de la licence souscrite par votre établissement. Toute autre reproduction ou représentation, en tout ou partie, sous quelque forme et de quelque manière que ce soit, est interdite sauf accord préalable et écrit de l'éditeur, en dehors des cas prévus par la législation en vigueur en France. Il est précisé que son stockage dans une base de données est également interdit. 


\title{
Ann Laura Stoler: La chair de l'empire. Savoirs intimes et pouvoirs raciaux en régime colonial 1
}

\author{
Par Natalia Gerodetti et Agathe Besnard ${ }^{2}$
}

Cela valait la peine d'attendre la traduction française du livre d'Ann Laura Stoler! La chair de l'empire, présenté par Éric Fassin dans la collection qu'Elsa Dorlin dirige avec lui, Genre \&t Sexualité, se situe dans la continuation du précédent livre d'Ann Laura Stoler, Race and the Education of Desire (Race et éducation du désir) publié en 1995, où elle offrait une relecture de l'Histoire de la sexualité (1976) de Michel Foucault. La chair de l'empire est un recueil d'essais plus qu'un récit progressif: Ann Laura Stoler, anthropologue et historienne, s'intéresse depuis des années au rôle central de la construction de la race et des empires dans la formation des sexualités européennes et à l'intégration de la sexualité dans les études critiques coloniales. Nous allons présenter ici la traduction française du livre d'Ann Laura Stoler, parue en 2013, à laquelle elle a ajouté une postface mais où les quarante-et-une images, présentes dans la version anglaise de 2002 manquent, ce qui est regrettable, car elles permettaient une représentation mentale de la subjectivité domestique coloniale.

La chair de l'empire est un regroupement d'essais écrits durant une période de vingt ans, agencés de manière pertinente pour le lectorat et constituant une riche synthèse de la pensée d'Ann Laura Stoler. Elle se concentre sur les colonies européennes, avec une importance plus grande accordée aux colonies néerlandaises indiennes. L'éventail des archives françaises et néerlandaises qu'elle utilise est impressionnant, les références bibliographiques provenant de sources primaires et secondaires sont très nombreuses. Dans la traduction française, le choix éditorial a été d'enlever les références littéraires néerlandaises et bon nombre des références

1. Ann Laura Stoler (2013). La chair de l'empire. Savoirs intimes et pouvoirs raciaux en régime colonial. Paris: La Découverte, coll. Genre \&t sexualité, 299 pages. Traduction par Sébastien Roux. Édition originale: Carnal Knowledge and Imperial Power: Race and the Intimate in Colonial Rule (Berkeley: University of California Press, 2002).
2. Natalia Gerodetti est actuellement maîtresse de conférences en sociologie et chercheuse à Leeds Beckett University, Angleterre. Elle travaille sur les questions de la régulation discursive et spatiale de "genre et sexualité», ainsi que sur un projet concernant l'identité des migrantes jardinières. Agathe Besnard est étudiante en troisième année de sociologie à l'Université Paris-Sorbonne. 
anglaises, remplacées par des références françaises équivalentes. Le résultat est un livre qui conserve la rigueur théorique et méthodologique de l'auteure.

Comme Joan Scott, Ann Laura Stoler recommande une réévaluation des catégories historiquement créées. Elle met aussi en garde contre les suppositions classiques qui fondent l'interprétation des causes des divisions traditionnelles au sein des colonies, privilégiant, pour sa part, l'idée que l'ensemble des métropoles, ainsi que les relations conflictuelles au sein de l'espace domestique, étaient au cœur des règles microphysiques coloniales. Au-delà du fait que son livre nous fournit ainsi une méthode qui permet d'évaluer le rôle des historien·ne-s dans les études concernant le genre et la création de l'histoire coloniale, il mobilise des théories philosophiques complexes, surtout celles de Foucault, ainsi que des analyses aussi diverses que celles de Pierre Bourdieu, Franz Fanon, George Lakoff et James Scott.

Le livre débute par une préface de Fassin, dans laquelle est retracé le parcours biographique et académique d'Ann Laura Stoler, pour celles et ceux qui ne connaîtraient pas bien son travail. Partant du métier d'ethnologue pour arriver au développement d'une approche plus anthropologique inspirée d'un point de vue marxiste, les interrogations d'Ann Laura Stoler sur les colonies l'ont menée d'Indonésie en Afrique du Sud, en passant par l'Inde et l'Algérie. Son intérêt se centre sur la formation impériale plutôt que sur la production d'explications historiques. Elle s'intéresse aux règles régissant les relations sexuelles et familiales des Indonésien-ne-s néerlandais·es et leur rôle fondateur dans le projet impérial.

Les trois premiers chapitres sont, en fait, trois essais classiques réimprimés qui retracent l'affection de l'auteure pour ce sujet depuis les années 1980. Ils présentent son intérêt théorique et historique pour les thématiques coloniales dans lesquelles le racisme était fondé sur une logique de classe qui ne concernait pas uniquement les "natifs" et les "natives", mais aussi les blanc·he.s démuni-e.s menaçant la morphologie des différences coloniales. Les inégalités de genre structuraient la formation des racismes coloniaux et des autorités impériales, particulièrement au sein de la sphère domestique. Les premiers chapitres exposent donc la composition du livre, les idées de l'auteure à propos de l'intimité au sein de la structure coloniale, ainsi que les théories et le travail académique associés aux études coloniales.

Dans le premier (intitulé «Introduction. Généalogies de l'intime. Dynamiques des études coloniales»), Ann Laura Stoler reprend la proposition d'Edward Said et sa notion d'orientalisme pour montrer comment l'Occident produit cette notion au travers de stratégies scientifiques et politiques. Les chapitres suivants fournissent de nombreuses illustrations de la matière complexe à laquelle l'auteure a dû faire face en s'intéressant aux dossiers judiciaires, aux lois portant sur le mariage, aux mouvements 
politiques, aux pratiques en matière d'éducation, ainsi qu'à d'autres facteurs qui participent à la constitution des identités coloniales. Elle développe l'idée que les catégories de colonisateur·trice et de colonisé·e ont été établies à travers le contrôle sexuel et la gouvernance des relations sexuelles qui délimitait non seulement le pouvoir domestique, mais créait aussi les frontières publiques, toujours par rapport à l'origine ethnique. Dans le deuxième chapitre, où elle reprend le titre de son livre, Ann Laura Stoler explore l'origine, la classe et le genre au sein des sociétés coloniales et postcoloniales. Elle examine comment la sexualité, le genre, l'origine - incluant le métissage -, l'éducation et l'abandon des enfants, le viol, la moralité et l'eugénisme étaient des idées structurantes à la fois dans les populations européennes et dans les populations indigènes. Elle montre que l'accès à la sexualité et les arrangements au sein du ménage n'étaient pas seulement privés et intimes, mais au contraire centraux dans la formation des structures de pouvoir. Le troisième chapitre explore plusieurs questions sur le métissage afin d'examiner comment les discours sur l'intégration et l'exclusion étaient déployés pour définir l'identité nationale et la citoyenneté.

Les essais plus récents (dès le quatrième chapitre) se focalisent sur la garde des enfants et les manières informelles d'enseigner la sensibilité aux normes coloniales. Le rôle des domestiques au sein de la sphère privée était essentiel, car même "si leur présence transgressait les différences de genre, de classe et de race, ils n'en étaient pas moins les gardiens subalternes du privé» (p. 186). Ann Laura Stoler présente aussi "une lecture coloniale de Foucault" où elle s'inspire de ses textes pour penser les catégories du corps bourgeois et le soi racial, en insistant sur le fait que la race est aussi fondamentale à la formation des États-nations modernes que la sexualité. Pour elle, une compréhension de la bourgeoisie n'est pas possible sans son inscription coloniale.

Dans un chapitre en collaboration avec Karen Strassler, la sphère familiale et domestique est traitée par le biais des mémoires des servantes javanaises et de la place du colonialisme dans leur récit. C'est ici que nous en apprenons davantage, avec "une vue du dessous", sur les expériences des femmes et des hommes indonésiens qui travaillaient sous l'autorité coloniale. Ces sentiments coloniaux et ces mémoires tactiles dans le contexte familial permettent de distinguer les similarités et les différences entre le contexte public et le contexte privé.

La chair de l'empire est un ouvrage percutant, doté d'une analyse condensée qui s'appuie sur des détails historiques et ethnographiques fondamentaux. Le livre s'achève par une claire invitation à mener plus de travaux portant sur la mémoire coloniale et à faire du colonial un sujet d'analyse. Avant tout, il est un appel à aller au-delà des catégories présumées et soulève des questions importantes pour les études sur le colonialisme, la sexualité et le genre, tant au passé qu'au présent. L'auteure estime impératif de développer davantage de critiques qui permettent de 
comprendre comment les formations impériales apparaissent et maintiennent leur pouvoir. Dans son œuvre, le passé rattrape le présent et viceversa. De plus, comme le dit Fassin dans la préface, l'œuvre d'Ann Laura Stoler apparaît "comme une réponse non seulement aux dénonciations rituelles de la (repentance, selon lesquelles l'histoire doit rester à sa place, c'est-à-dire dans le passé, mais aussi aux polémiques à répétition dans le monde scientifique français [...] contre les études (postcoloniales ', accusées d'écraser dans un même terme passé et présent» (p. 17). 